\title{
Atomic Scale Simulations of Relationship between Macroscopic Mechanical Properties and Microscopic Defect Evolution in Ultrafine-grained Metals
}

\author{
Tomohito Tsuru ${ }^{1, *}$, Yoshiteru Aoyagi ${ }^{2}$ and Tomotsugu Shimokawa ${ }^{3}$ \\ ${ }^{1}$ Nuclear Science and Engineering Center, Japan Atomic Energy Agency, Tokai-mura, Ibaraki 319-1195, Japan \\ ${ }^{2}$ Graduate School of Engineering, Tohoku University, Sendai 980-8579, Japan \\ ${ }^{3}$ Faculty of Mechanical Engineering, Kanazawa University, Kanazawa 920-1192, Japan
}

\begin{abstract}
The effects of grain size and intragranular dislocation on yield mechanism and subsequent plastic deformation in ultrafine-grained (UFG) $\mathrm{Al}$ and $\mathrm{Cu}$ were investigated by large-scale atomic simulations. Polycrystalline atomic models with and without intragranular dislocation sources were used to elucidate the relationship between mechanical properties and defect texture. It is found that the intragranular dislocation plays a significant role in both incipient yield and grain boundary mediated dislocation nucleation. In addition UFG Cu yields earlier than UFG Al because partial dislocations in $\mathrm{Cu}$ are more likely to activate from grain boundaries, where the partial dislocation leaves deformation twin and secondary dislocation tends to move on twin boundary accompanied by the shift of twin boundary plane. [doi:10.2320/matertrans.MH201515]
\end{abstract}

(Received April 27, 2016; Accepted May 26, 2016; Published June 24, 2016)

Keywords: $\quad$ ultrafine-grained metals, grain size, intragranular dislocation, twin boundary, atomistic simulations

\section{Introduction}

Plastic deformation of conventional coarse grained (CG) metals (grain size $d>1 \mu \mathrm{m}$ ) is generally determined by the average quantity of collective motion of dislocations. The Hall-Petch relation ${ }^{1,2)}$ has been used for an effective guide for material design, where the interaction between piled-up dislocations and grain boundaries (GB) plays a dominant role in strengthening mechanism of materials. Conversely, nanocrystalline (NC) metals $(d<20 \mathrm{~nm})$ deforms by means of GB-mediated plasticity. The material strength of NC metals decreases with decreasing grain size, which is generally known as the inverse H-P effect ${ }^{3,4)}$. Therefore, deformation mechanism and the major agent of plastic deformation differ depending on grain size.

Recently extreme grain refinement techniques were developed through severe plastic deformation processing ${ }^{5-8)}$ which enable to produce ultrafine-grained (UFG) metals $(100 \mathrm{~nm}<$ $d<1 \mu \mathrm{m})$ in bulk, so many unique and excellent mechanical properties have been proposed for the last dozen years ${ }^{9-13)}$. However, their plastic deformation cannot simply be explained by the well-known mechanisms for CG and NC metals. "Hardening by annealing and softening by deformation" is one of the distinguishing characteristics of UFG metals ${ }^{9)}$. Here the dislocation density was reduced to one-third by annealing $\left(1.33 \times 10^{14}\right.$ to $\left.0.53 \times 10^{14} 1 / \mathrm{m}^{2}\right)$ while slight coarsening occurred during annealing for the lamellar boundary spacing; $180 \mathrm{~nm}$ to $225 \mathrm{~nm}$ and for the interconnecting boundary spacing; $600 \mathrm{~nm}$ to $680 \mathrm{~nm}$. A simple estimation indicates that the average number of dislocations within each grain is less than 10 and decreases by half after annealing. Consequently, this slight difference of the number of intragranular dislocations seems to influence the mechanical properties of UFG metals.

What is the dominant plastic deformation mechanism of UFG metals? Unlike conventional CG metals, UFG metals have a dramatic increase in the volume fraction of the GB

*Corresponding author, E-mail: tsuru.tomohito@jaea.go.jp region. Furthermore, as mentioned above, the number of dislocations within grain region decreases significantly even if the dislocation density is the same as CG metals; hence, plastic deformation is no longer predicted by the average quantity of collective motion of dislocations in each grain region. In other words, the grain size of UFG metals is too small to include a sufficient number of intragranular dislocations in a grain region in comparison with CG metals, while unlike the NC metals, the grain size is too large for GB migration, sliding and diffusion processes to fully account for all the plastic strain. Thus, GB-mediated dislocation behavior like dislocation emission from GB probably plays a significant role in plastic deformation of UFG metals and a few numbers of dislocations in a grain could influence the GB-mediated dislocation nucleation behavior.

Atomistic simulations have been applied to reconstruct specific GB structures. Dislocation and twin nucleation process were investigated using pseudo three-dimensional models ${ }^{14-16)}$. Our previous study has revealed that pre-existing dislocations as well as the nucleation process play more important role in deformation in UFG metals ${ }^{17}$ ). Therefore fully three-dimensional model is required to consider the possible activation of all slip systems of pre-existing dislocations. However, there are few computational studies to understand the anomalous mechanical properties of UFG metals taking internal defect structures into account.

In the present study, large-scale atomistic simulations were implemented to elucidate the effects of grain size and intragranular dislocations on mechanical properties of UFG metals. Deformation behavior of polycrystalline models with different grain size with/without intragranular dislocations was investigated through tensile deformation. Subsequently the effect of stacking fault (SF) energy on GB-mediated dislocation nucleation behavior was examined by comparative analysis of $\mathrm{Al}$ and $\mathrm{Cu}$.

\section{Computational Method}

Three-dimensional polycrystalline atomic models of 
face-centered-cubic (fcc) $\mathrm{Al}$ and $\mathrm{Cu}$ were constructed using Voronoi polyhedral division. The atomic models have different grain size according to $4.5-33.6 \mathrm{~nm}$ for $\mathrm{Al}$ and 4.0 $30.0 \mathrm{~nm}$ for $\mathrm{Cu}$, respectively, and the simulation box is 2.7 times larger than the average grain size. When the relative position of the Voronoi center is fixed regardless of the simulation box, all atomic models have the same morphology except for grain size. Atomistic models of polycrystalline were considered to be cubic, containing 15 grains in the present study. The number of atoms of the largest model is approximately 43,000,000 atoms. Pre-existing dislocation sources were treated, where a single crystal including a Frank-Read source, several voids and interstitials were prepared beforehand, and uniaxial loading were applied to the single crystalline model. The Frank-Read source was activated under loading and the dislocation density increased due to the dislocation multiplication. In addition to multiplication mechanism, voids and interstitials act as the pinning of dislocation gliding so that the dislocations get tangled and the dislocation density increases significantly. After the dislocation density reached $10^{16} \mathrm{~m}^{-2}$ order, the uniaxial strain was removed quickly. Here the dislocation density was estimated by the relative number of high-energy atoms which corresponds to dislocation line, since the length of a dislocation and the number of those atoms constitute dislocations have a one-on-one relationship. Note that the dislocation density discussed in this study is a rough estimation and that relative change is our interest. Accordingly, a single crystal including highly-tangled dislocation was prepared. Two single crystals (i.e. "defect-free" model and "high-dislocation-density" model) were embedded into grain regions, and consequently each polycrystal with the same grain size has the different internal defect structure. Similarly, the atomic fraction of GB region can be estimated by counting the atoms having the different energy from the defect-free region and the ratio of these atoms to total number of atoms of the simulation box is the atomic fraction as discussed below.

A hybrid parallelized molecular dynamics (MD) code with efficient communication and I/O techniques using MPI-IO was developed and applied to treat the collective defect mechanics within the large-scale atomic models. MPI-IO was applied to the visualization software ATOMEYE ${ }^{18)}$, where the defect structures of presented models including dislocations and GBs, were visualized with the parallelized technique. The embedded atom method (EAM) potential proposed by Mishin et al. was employed for the interatomic interactions of $\mathrm{Al}$ and $\mathrm{Cu}$ atoms ${ }^{19,20)}$. Stable configurations of polycrystals including various GB structures were obtained considering finite temperature molecular dynamics and molecular statics with conjugate gradient energy minimization.

Uniaxial tension was then applied to the atomic models, where a strain increment $\Delta \varepsilon_{z z}$ of $1.0 \times 10^{-3}$ was uniformly applied to the simulation box. After applying tensile strain, the simulation box was relaxed so that the normal stress components perpendicular to the load direction were controlled to be zero while at the same time all atoms were relaxed by molecular statics simulations. Here, stress component can be evaluated based on virial theorem ${ }^{21)}$. Thus quasi-equilibrium configuration under the deformation at $0 \mathrm{~K}$ was obtained. Triaxial periodic boundary conditions were satisfied during the uniaxial tensile loading. The atomic system under given strain was optimized by sufficient relaxation steps, and fully relaxed configuration for each condition was defined as that when the energy norms of all atoms converged at $0.1 \mathrm{eV} / \AA$ or conjugate gradient step reaches 4000 . This cycle was repeated until the maximum tensile strain reached $8 \%$.

DFT calculations were carried out to verify the validity of the twin boundary (TB) and stacking fault (SF) energy evaluated by EAM potential, where the Vienna Ab initio simulation package (VASP) ${ }^{22,23)}$ was used with the Perdew-BurkeErnzerhof formulation of the generalized gradient approximation to the exchange-correlation density functional ${ }^{24)}$. The plane-wave energy cutoff was set at $400 \mathrm{eV}$. Atomic models including 24 layer along $z[111]$ direction as well as $x[\overline{1} 10]$ and $y[\overline{1} \overline{1} 2]$ units are used for both TB and SF models. The Brillouin-zone $16 \times 9 \times 1 k$-point samplings were chosen using the Monkhorst-Pack algorithm ${ }^{25}$.

\section{Results and Discussions}

\subsection{Internal texture of atomic models}

The atomic fraction of GB region and dislocation density estimated from atomic models are summarized in Fig. 1(a) and (b), respectively. The atomic fraction is significantly increases with decreasing grain size which makes little difference between $\mathrm{Al}$ and $\mathrm{Cu}$ when it is normalized by the unit length of the crystal lattice (i.e. lattice constant: $a_{0}$ ). On the other hand, the dislocation density decreases with decreasing grain size. Supposing that a similarity is maintained in that the ratio of the average length of dislocation to grain size is fixed, the dislocation density increases by a factor of $k^{2}$ when
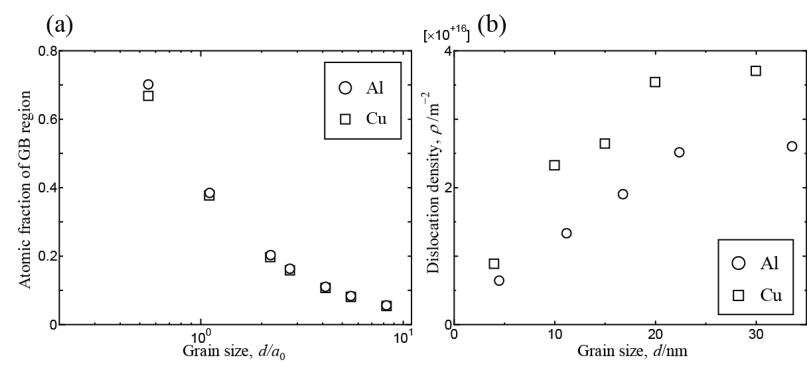

(c)
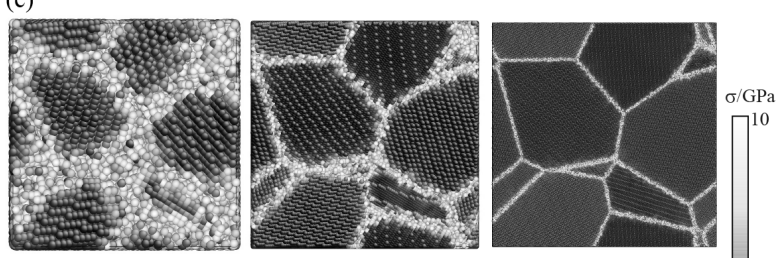

(d)
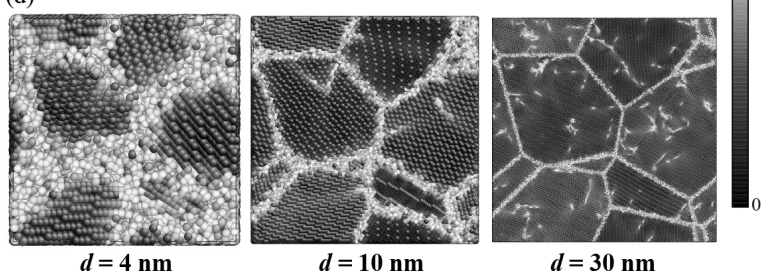

Fig. 1 Microstructure of atomic UFG models. (a) Relationship between atomic fraction of GB region and grain size. (b) Relationship between estimated dislocation density and grain size. (c) Mises stress distribution of dislocation-free UFG Cu models with different grain size. (d) Same as (c) but for high-dislocation-density models. 
grain size decreases by a factor of $1 / k$, where $k$ is an arbitrary real number. More specifically, the dislocation density of the UFG metal seems much higher than that of coarse-grained metals even when the ratio of the average distance between dislocations to grain size is constant. However, our result shows an opposite trend because GB region has an ability to absorb lattice defect such as dislocation. A large volume fraction of GB corresponding to the atomic fraction absorbs a number of dislocations into GB as shown in Fig. 1(a). In addition the dislocation density varies between $\mathrm{Al}$ and $\mathrm{Cu}$ in association with the difference of dislocation core structure, that is, partial dislocations in $\mathrm{Cu}$ are easily tangled which prevents from annihilating and absorbing. Atomic images of polycrystalline models without and with dislocations for different grain size $(d=4,10,30 \mathrm{~nm})$ in $\mathrm{Cu}$ are shown in Fig. 1(c) and (d), respectively, where defect structures are visualized according to the atomic Mises stress which highlights the dislocation core and the heterogeneous GB region all together. There are few dislocations in grain region in case of $d=4 \mathrm{~nm}$ due to the absorption of pre-existing dislocations into GB region while a number of dislocations are left as grain size becomes larger. It is also confirmed that $\mathrm{Cu}$ polycrystals have more dislocations in grain region than $\mathrm{Al}$ polycrystals.

\subsection{Deformation process of UFG $\mathrm{Al}$ and $\mathrm{Cu}$}

The effects of initial dislocation density and grain size on deformation behavior were investigated using atomic models prepared above. These models would be a good analogy for "as-deformed" and "annealed" UFG specimens in the experiments, i.e. the dislocation density of as-deformed specimen is relatively higher than that of annealed one ${ }^{9}$. Typical stressstrain curves under tensile deformation for different grain size $\left(d=1.1 a_{0}, 2.8 a_{0}\right.$ and $\left.8.3 a_{0}\right)$ are shown in Fig. 2, where the effect of internal texture are depicted as well as grain size dependence. It should be mentioned that the yield stresses calculated using above atomic models are used to capture the qualitative nature as the number of grain within a simulation box is insufficient for a statistical amount due to the computational cost. When the grain size is smaller than $10 \mathrm{~nm}$, there is little difference in stress-strain curves for both $\mathrm{Al}$ and $\mathrm{Cu}$ because two models have almost the same initial dislocation density. Pre-existing dislocation sources are absorbed into GB region in this type of small grain $(d<10 \mathrm{~nm})$. A remarkable difference between dislocation-free and high-dislocation-density models was found in Fig. 2 as the grain size increases. This fact clearly indicates that pre-existing dislocation sources within the grain region are activated prior to macroscopic yield, where the initial local maximum point is defined as the yield stress $\left(\sigma_{\mathrm{Y}}\right)$. As mentioned above, the yield stress varies according to the initial dislocation density since the incipient plastic deformation is caused by the activation of pre-existing dislocations. The tendency of stressstrain curves for high-dislocation-density models is similar to continuous yielding behavior observed in coarse-grained metals with fcc structure, where the yield point is ambiguous during elastic-plastic deformation. On the other hand, the yield drop becomes conspicuous in case of dislocation-free model. Therefore, the yield stress of UFG metals strongly depends on the initial dislocation density. Dislocations no lon-
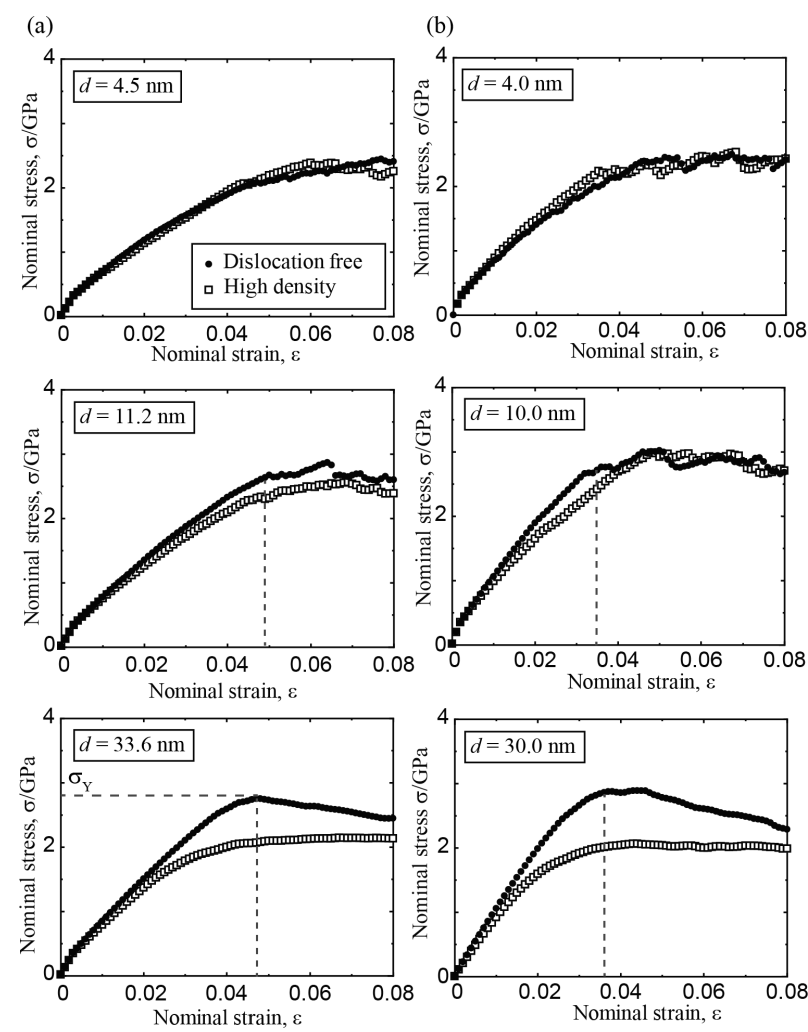

Fig. 2 Stress strain curves under tension for different grain size in (a) UFG $\mathrm{Al}$ and (b) UFG Cu.

ger exist homogeneously as the initial dislocation density decreases. There are only a few dislocation sources within a grain region which prevent from continuous yield. By extracting the yield stress from all stress-strain curves, relationships between the yield stress and grain size for different dislocation density are summarized in Fig. 3. It should be noted in high dislocation density model that the tendency of the effect of grain size on yield stress changes around $d=10 \mathrm{~nm}$; the yield stress increases with decreasing grain size when $d>$ $10 \mathrm{~nm}$ while the stress decreases when $d<10 \mathrm{~nm}$. This trend is a universal feature for all materials known as inverse HallPetch relation ${ }^{1,2)}$ where plastic deformation evolves through GB rotation or sliding. On the other hand, this trend cannot be seen in dislocation-free model. While inverse Hall-Petch mechanism works for dislocation-free models when $d<$ $10 \mathrm{~nm}$, conventional Hall-Petch relation is not applicable to dislocation-free model because there are no dislocation sources within grain region. Furthermore, the mechanism of dislocation nucleation from GB does not change significantly even when grain size becomes larger. Here, the ideal tensile strength of polycrystal can be estimated by the analogy of Taylor factor on the assumption that each grain yield when the maximum resolved shear stress reaches the ideal shear strength. The ideal strengths are found to be 9.8 and $8.9 \mathrm{GPa}$ for $\mathrm{Al}$ and $\mathrm{Cu}$, where the ideal shear strengths were 3.19 and $2.86 \mathrm{GPa}$ for employed EAM potentials of $\mathrm{Al}$ and $\mathrm{Cu}$ which relatively agree with density functional theory (DFT) calculations ${ }^{26)}$. The tensile stress of polycrystals should not only exceed this upper limit but be much lower than the limit, because the critical shear stress for dislocation nucleation at GB must be lower than ideal shear strength. The critical shear 
(a)

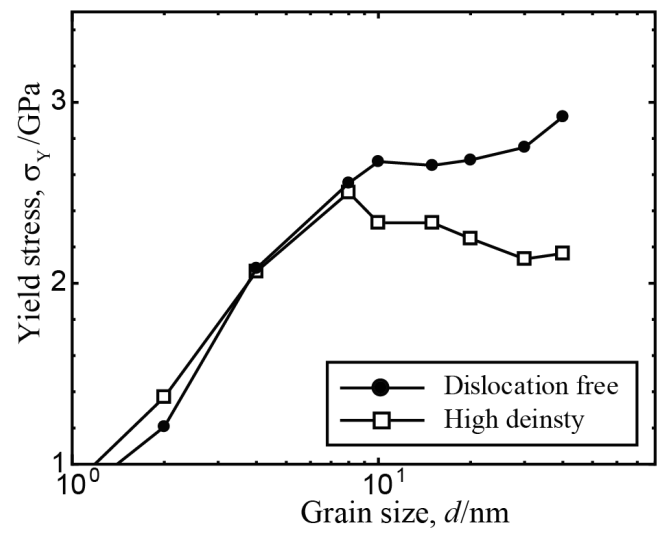

(b)

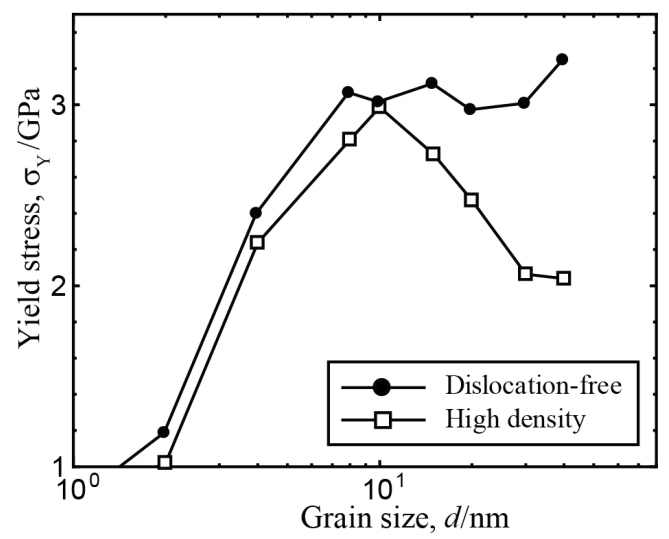

Fig. 3 Relationship between yield stress and grain size in (a) UFG Al and (b) UFG Cu.

stress for nucleation at GB was indeed around 1 to $2 \mathrm{GPa}$. The dislocation nucleation at GB is independent of grain size because this nucleation event is only localized around GB. The yield stresses of dislocation-free models are expected to saturate a certain limit depending on the local characteristics around GB. Here yield behavior in cases of dislocation-free and high-dislocation-density models for UFG-Cu $(d=10 \mathrm{~nm})$ are shown in Fig. 4(a) and (b). In this figure only atoms with centrosymmetry (CS) parameter ${ }^{27)}$ in the range from 0.001 to 0.1 are extracted and colored to emphasize defect structures such as dislocation and GB. Configurations of initial and before/after yield are taken as typical defect evolution. GB-mediated defect nucleation is observed in Fig. 4(a) as discussed above. On the other hand, incipient plastic deformation occurs as the activation of intragranular dislocation sources as seen in Fig. 4(b) $(\varepsilon=0.00 \sim 0.03)$. This local yield process corresponds to the reduction in gradient of elastic-plastic stress-strain behavior before the macroscopic yield of high density models shown in Fig. 2(b). Additionally more defects are observed at $\varepsilon=0.06$ despite the same applied strain; GB-mediated defects are likely to activate from GB. The processes of dislocation absorption into GB and subsequent defect nucleation are shown in Fig. 4(c). The residual component of the Burgers vector would facilitate the subsequent secondary defect nucleation at GB after intragranular dislocations absorb into GB. Thus high-dislocation-density model or as-deformed specimen provides continuous yielding behavior. If these GB-mediated defects influence the macroscopic (a)
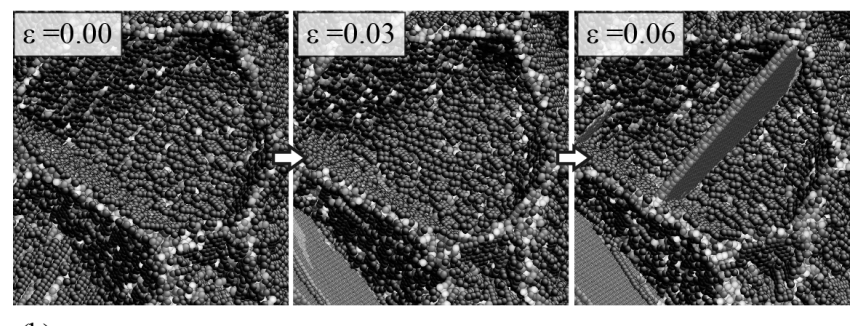

(b)

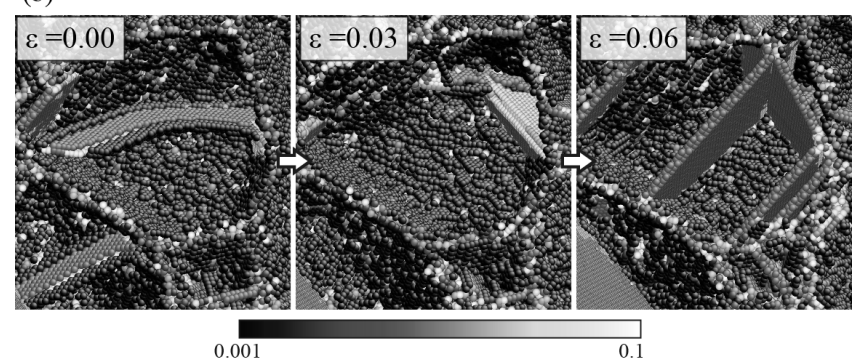

(c)

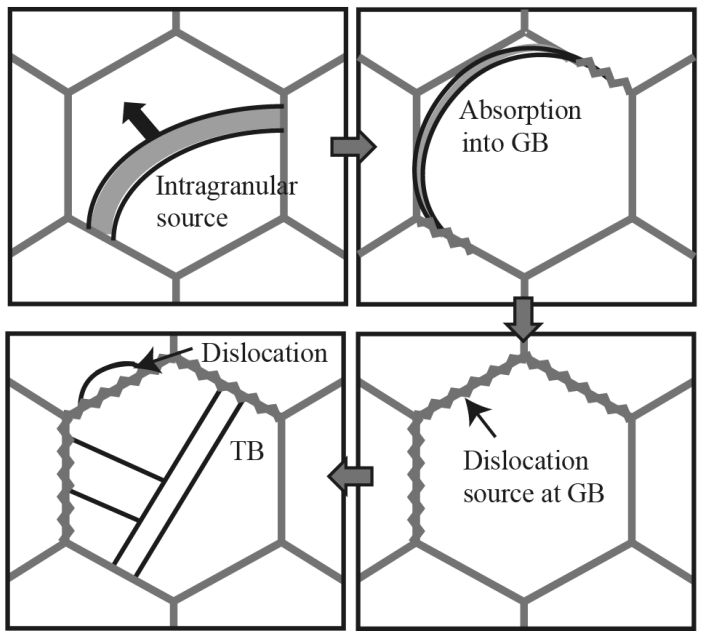

Fig. 4 Atomic images of defect structure of initial configuration $(\varepsilon=0.00)$ and before/after yield $(\varepsilon=0.03,0.06)$ for UFG Cu with $d=10 \mathrm{~nm}$, which are visualized by CS parameter in the given range. (a) Dislocation-free model. (b) High-dislocation-density model. (c) Schematic diagram of dislocation absorption and secondary defect nucleation at GB.

yield stress according to Fig. 3, the critical stress for the defect nucleation from GB should show grain size dependence. Kato and Wang proposed a thermally activated dislocation process $^{28-30)}$, where deformation of UFG metals was determined by the depinning and bow-out process of dislocations at GB. According to this model, although our simulation was performed at $0 \mathrm{~K}$, grain size dependence in Fig. 3 can be explained by the distance between two pinning at GB which is directly proportional to grain size.

\subsection{Formation of multiple deformation twins}

It is approximately expected within the framework of linear elastic and classical dislocation theory that the ideal strength or yield stress correlates linearly with elastic modulus. It has been known that ideal shear strength is no longer expected linearly but influenced by electronic binding ${ }^{26)}$. As well as ideal strength of $\mathrm{Al}$ and $\mathrm{Cu}$, the yield stresses calculated in the present study disagree with the classical theory. The yield stress of UFG-Al is as high as that of UFG-Cu even 


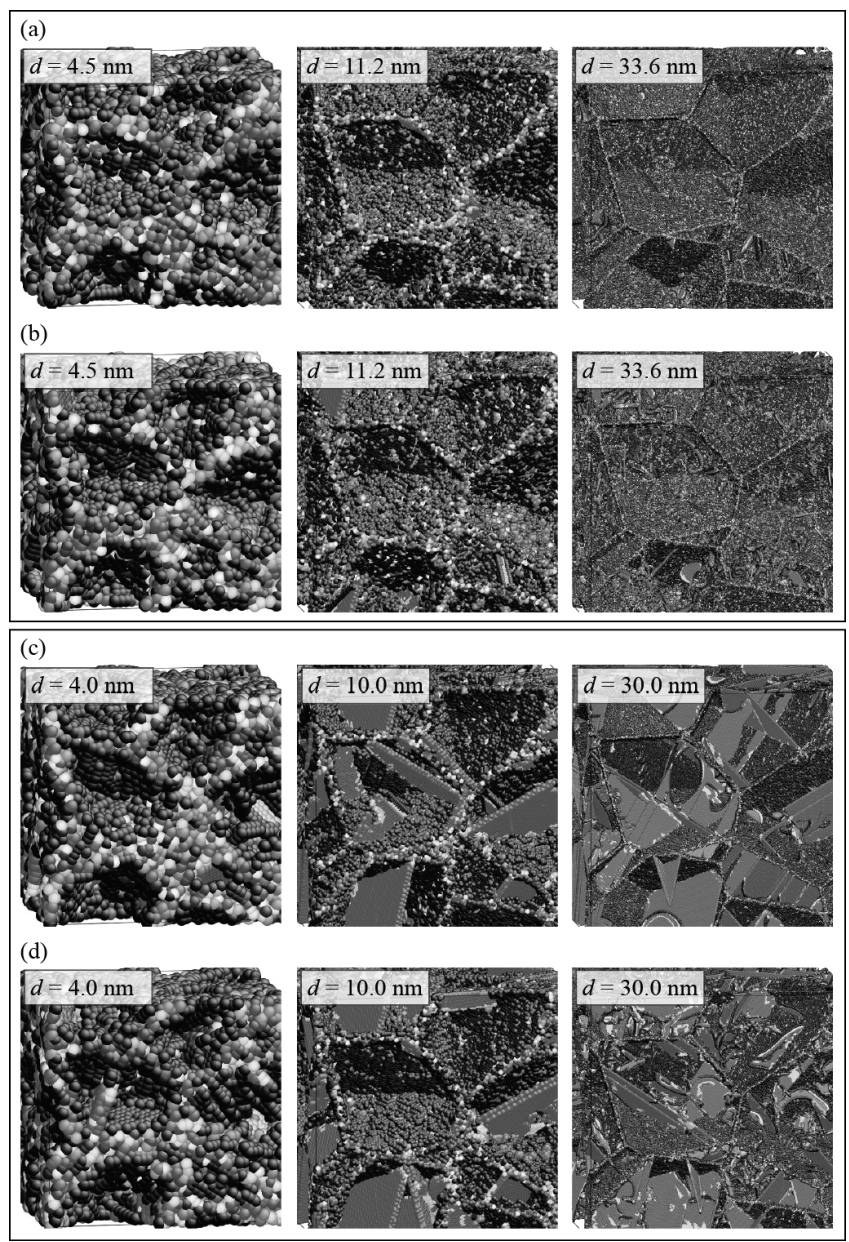

Fig. 5 Atomic images of defect structure after yield point $(\varepsilon=0.06)$. (a) Dislocation-free UFG Al. (b) High-dislocation-density UFG Al. (c) Same as (a) but for $\mathrm{Cu}$. (d) Same as (b) but for $\mathrm{Cu}$.

though the shear modulus of $\mathrm{Cu}$ is 1.5 times higher than that of Al. Nucleation mechanism of defect structures is essential for understanding the difference in yield stress.

Internal defect structures of $\mathrm{Al}$ and $\mathrm{Cu}$ during tensile deformation were visualized in Fig. 5, where the effects of grain size and initial dislocation density after yield point $(\varepsilon=0.06)$ are compared. When grain size is smaller than $10 \mathrm{~nm}$, there is little difference in deformation texture regardless of initial dislocation density. It is confirmed in both $\mathrm{Al}$ and $\mathrm{Cu}$ that GB-mediated plasticity is dominant under tensile deformation. Accordingly there is no significant distinction between $\mathrm{Al}$ and $\mathrm{Cu}$. As grain size becomes larger, however, deformation texture shows clear difference, i.e. more dislocations are nucleated in $\mathrm{Cu}$ despite the same strain. Activation of intragranular dislocation sources embedded in high-dislocation-density models is generally determined by the simple Orowan mechanism, and they were activated much earlier than the macroscopic yield point in both $\mathrm{Al}$ and $\mathrm{Cu}$, where the bow-out dislocations produce little plastic strain due to the limited of range of motion within grain region. Macroscopic yield occurs when a number of dislocations are activated. The dislocation density increases gradually after the macroscopic yield accompanied with dislocation nucleation at the GB. Here, in contrast to intragranular sources, the activation of intergranular sources differs substantially between $\mathrm{Al}$ and $\mathrm{Cu}$ (a)
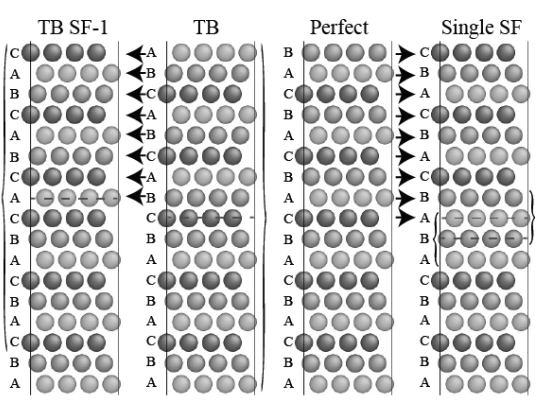

Double SF-1 Double SF-2 $\rightarrow$ A $10009 \rightarrow$ a 10009 $\rightarrow \mathrm{B} 0000 \rightarrow \rightarrow_{\mathrm{B}}^{\mathrm{C}} 0000$ $\rightarrow \mathrm{A} 0000 \rightarrow \mathrm{A} 0000$ B 0000 (1000 0-9 a 0000 a l 0000 ( 10000 A 0000 1000 c0000 р000 в 0000

(b)
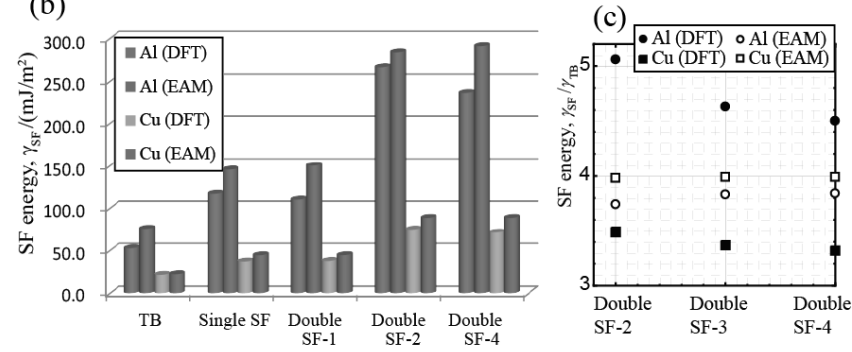

(d)

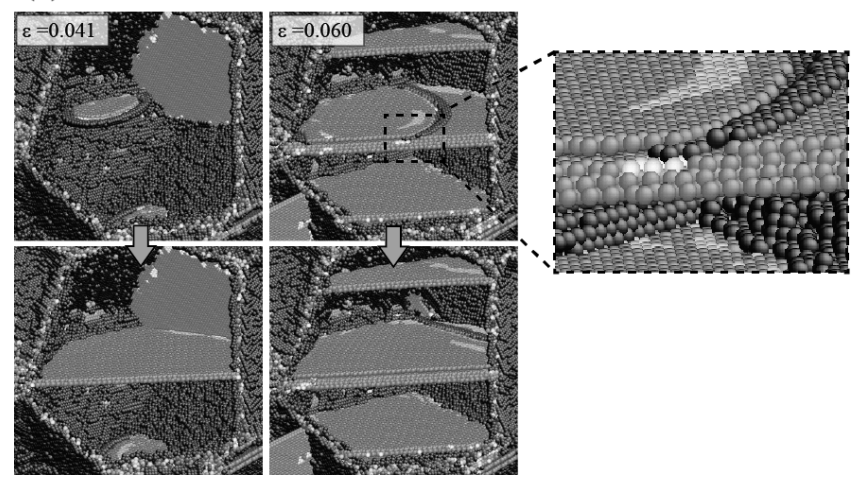

Fig. 6 TB and SF formation accompanied with a secondary SF plane. (a) Various stacking states of TB and SF. (b) TB and SF energies in $\mathrm{Al}$ and Cu. (c) Relative energies of double SF to TB energy in $\mathrm{Al}$ and $\mathrm{Cu}$. (d) Atomic images of initial SF and DSC dislocation nucleations.

due to the difference of SF energy. Extended dislocations are generally more stable in $\mathrm{Cu}$ due to the low SF energy, which can be reproduced by employed EAM potential. In addition partial dislocations are likely to activate from GB because of the small Burgers vector and consequently plastic deformation of $\mathrm{Cu}$ starts earlier than that of $\mathrm{Al}$. As the result of easy dislocation nucleation from GB, as shown in Fig. 2, $\mathrm{Cu}$ yields relatively earlier than $\mathrm{Al}$ reflecting the microscopic texture.

There are a number of deformation twin nucleated in $\mathrm{Cu}$ according to low SF energy. The formation of TB, single SF and double SF placed at different distance considered in Fig. 6, where atomic images of the TB and single/double SF staking and their formation energies are depicted in Fig. 6(a) and (b), respectively. In Fig. 6(a) dashed lines indicate SF layers. The SF energies were evaluated by both EAM potential and DFT. The fully relaxed configurations were obtained by relaxing atomic displacement along [111] direction. It is confirmed in Fig. 6(b) TB is less likely nucleated in $\mathrm{Al}$ due to the higher TB energy. The SF energy of a single SF is nearly twice as high as TB energy because the single SF includes multi TB layers across the SF plane. TB energy evaluated by EAM potential are indeed almost twice $\left(\gamma_{\mathrm{SF}}=1.92 \gamma_{\mathrm{TB}}\right.$ for $\mathrm{Al}$ 
and $\gamma_{\mathrm{SF}}=1.99 \gamma_{\mathrm{TB}}$ for $\mathrm{Cu}$, respectively). Here it should be noted that the SF energy of $\mathrm{Al}$ is higher than twice the TB energy $\left(\gamma_{\mathrm{SF}}=2.22 \gamma_{\mathrm{TB}}\right)$ while that of $\mathrm{Cu}$ is lower $\left(\gamma_{\mathrm{SF}}=1.72\right.$ $\left.\gamma_{\mathrm{TB}}\right)$ when evaluated by DFT. Double SF-1 and TB SF-1 models correspond to another SF layer generated on (111) adjacent to the initial SF and TB plane, and double SF-2 and SF-4 have another SF on 2 and 4 layers away from initial SF plane. TB SF-1 is identical to initial TB except that TB plane shifts its position and therefore the energy is completely the same as initial TB stacking. Similarly there is little difference in SF energy between double SF-1 and single SF because the double SF-1 changes the position of one side of SF plane ${ }^{31)}$. Except for double SF-1, however, the SF energy of double SF models is as high or higher than twice the SF energy according to the number of equivalent TB plane. The SF energies of double SF-2, 3 and 4 relative to TB energy are shown in Fig. 6(c). It is seen that the energies evaluated by DFT are strongly influenced by spacing layers. DFT results show the energies decrease with distance from initial SF plane while there is little effect on spacing in energies evaluated by EAM potential. According to these characteristics of EAM potential for both $\mathrm{Al}$ and $\mathrm{Cu}$, secondary slip is more likely to occur on pre-existing SF plane which is generally known as displacement shift complete (DSC) lattice dislocation. Additionally, the DSC lattice dislocation is nucleated by the interaction between TB and dislocations on other slip plane and that the energy barrier of slip motion of DSC lattice plane along the lowest energy path is lower than that of $<\overline{1} \overline{1} 2>(111)$ slip in perfect crystal $^{32,33)}$. A typical nucleation process of TB and DSC lattice dislocation are shown in Fig. 6(d). The reading partial moves to other side of GB without trailing partial, resulting in TB plane across each grain. It is confirmed that multiple slip is formed readily from GB and that the secondary dislocations are nucleated as DSC lattice dislocations, where the shift of TB plane is indeed observed during the slip of DSC lattice dislocation. Thus, in UFG metals the deformation twin is more likely to be generated and left within the grain region, and these TBs grow easily through either multiple slip or interaction between TB and dislocation.

\section{Conclusion}

The effects of grain size and intragranular dislocations on deformation behavior of UFG $\mathrm{Al}$ and $\mathrm{Cu}$ were investigated using large-scale atomic simulations. The dislocation density decreases with decreasing grain size due to the absorption into GB because the volume fraction of GB region increases. The effect of initial dislocation density on yield stress becomes significant when grain size is greater than $10 \mathrm{~nm}$, where intragranular dislocations are activated prior to macroscopic yield. Additionally GB-mediated defect nucleation was triggered significantly by residual Burgers vector of absorbed dislocation.

The influence of SF energy on GB-mediated nucleation process was investigated by comparing $\mathrm{Al}$ and $\mathrm{Cu}$. UFG $\mathrm{Cu}$ yields earlier than UFG Al due to the difference in dislocation core structure, i.e. partial dislocation of $\mathrm{Cu}$ is more likely to activate from GB. Deformation twins tend to be nucleated in UFG $\mathrm{Cu}$ according to low SF energy, where leading partials reaches other side of GB without trailing partials. Once deformation twin was generated, the secondary dislocation becomes more stable when it moves on TB plane which is the deformation-induced growth mechanism of TB.

\section{Acknowledgments}

The authors acknowledge financial support from MEXT KAKENHI Grant number 22102007 (Grants-in-Aid for Scientific Research on Innovative Areas, "Bulk Nanostructured Metals”). T.T acknowledges financial support from Collaborative Research Based on Industrial Demand of JST and technical support of parallel computing provided by the Center for Computational Science and e-Systems at JAEA.

\section{REFERENCES}

1) E.O. Hall: Proc. Phys. Soc. Lond. B 64 (1951) 747-753.

2) N.J. Petch: J. Iron Steel Inst. 174 (1953) 25-28.

3) H. Chokshi, A. Rosen, J. Karch and H. Gleiter: Scr. Metall. 23 (1989) 1679-1684.

4) G.E. Fougere, J.R. Weertman, R.W. Siegel and S. Kim: Scr. Metall. Mater. 26 (1992) 1879-1883.

5) Y. Iwahashi, Z. Horita, M. Nemoto, J. Wang and T.G. Langdon: Scr. Mater. 35 (1996) 143-146.

6) Y. Saito, H. Utsunomiya, N. Tsuji and T. Sakai: Acta Mater. 47 (1999) 579-583.

7) R.Z. Valiev, Y.V. Ivanisenko, E.F. Rauch and B. Baudelet: Acta Mater. 44 (1996) 4705-583.

8) S.H. Lee, Y. Saito, N. Tsuji, H. Utsunomiya and T. Sakai: Scr. Mater. 46 (2002) 281-285.

9) X. Huang, N. Hansen and N. Tsuji: Science 312 (2006) 249-251.

10) Y.M. Wang and E. Ma: Acta Mater. 52 (2004) 1699-1709.

11) Y. Kimura, T. Inoue, F. Yin and K. Tsuzaki: Science 320 (2008) 10571060 .

12) N. Tsuji, Y. Ito, Y. Saito and Y. Minamino: Scr. Mater. 47 (2002) 893899.

13) N. Kamikawa, X. Huang, N. Tsuji and N. Hansen: Acta Mater. 57 (2009) 4198-4208.

14) V. Yamakov, D. Wolf, M. Salazar, S.R. Phillpot and H. Gleiter: Acta Mater. 49 (2001) 2713-2722.

15) V. Yamakov, D. Wolf, S.R. Phillpot, A.K. Mukherjee and H. Gleiter: Nat. Mater. 1 (2002) 45-49.

16) H. Van Swygenhoven, P.M. Derlet and A.G. Froseth: Nat. Mater. 3 (2004) 399-403.

17) T. Tsuru, Y. Aoyagi, Y. Kaji and T. Shimokawa: Mater. Trans. 54 (2013) 1580-1586.

18) J. Li: Model. Simul. Mater. Sci. Eng. 11 (2003) 173-177.

19) Y. Mishin, D. Farkas, M.J. Mehl and D.A. Papaconstantopoulos: Phys. Rev. B 59 (1999) 3393

20) Y. Mishin, M.J. Mehl, D.A. Papaconstantopoulos, A.F. Voter and J.D Kress: Phys. Rev. B 63 (2001) 224106.

21) M. Zhou: Proc. R. Soc. Lond. A 459 (2003) 2347-2392.

22) G. Kresse and J. Hafner: Phys. Rev. B 47 (1993) 558-561.

23) G. Kresse and J. Furthmuller: Phys. Rev. B 54 (1996) 11169-11186.

24) J.P. Perdew, J.A. Chevary, S.H. Vosko, K.A. Jackson, M.R. Pederson, D.J. Singh and C. Fiolhais: Phys. Rev. B 46 (1992) 6671-6687.

25) H.J. Monkhorst and J.D. Pack: Phys. Rev. B 13 (1976) 5188-5192.

26) S. Ogata, J. Li and S. Yip: Science 298 (2002) 807-811.

27) C.L. Kelchner, S.J. Plimpton and J.C. Hamilton: Phys. Rev. B 58 (1998) 11085 .

28) M. Kato: Mater. Sci. Eng. A 516 (2009) 276-282

29) M. Kato: Mater. Trans. 56 (2015) 175-181.

30) Y.M. Wang, A.V. Hamza and E. Ma: Acta Mater. 54 (2006) 2715-2726.

31) S. Ogata, J. Li and S. Yip: Phys. Rev. B 71 (2005) 224102.

32) T. Tsuru, Y. Shibutani and Y. Kaji: Phys. Rev. B 79 (2009) 012104.

33) T. Tsuru, Y. Kaji, D. Matsunaka and Y. Shibutani: Phys. Rev. B 82 (2010) 024101. 